\title{
SARS CoV-2 Second Wave: Enlightening Nigerians About Latest SARS CoV-2 Variants and the Clinical Manifestation of the Viral Infection in Different Disease Conditions
}

\author{
Sunday Zeal Bala ${ }^{1, *}$, Miracle Livinus ${ }^{1}$, Madinat Hassan ${ }^{2}$, Solomon Bitrus ${ }^{3}$ \\ ${ }^{1}$ Biochemistry Department, Faculty of Basic Medical Science, Bayero University, Kano, Nigeria \\ ${ }^{2}$ Biology Department, Faculty of Science, Airforce Institute of Technology, Kaduna, Nigeria \\ ${ }^{3}$ Biology Department, Faculty of Science, Kaduna State University, Kaduna, Nigeria
}

Email address:

drealzeal@gmail.com (S. Z. Bala), livinusmiracle2009@yahoo.com (M. Livinus), m.hassan@afit.edu.ng (M. Hassan), bitsolo2007@gmail.com (S. Bitrus)

${ }^{*}$ Corresponding author

To cite this article:

Sunday Zeal Bala, Miracle Livinus, Madinat Hassan, Solomon Bitrus. SARS CoV-2 Second Wave: Enlightening Nigerians About Latest SARS CoV-2 Variants and the Clinical Manifestation of the Viral Infection in Different Disease Conditions. International Journal of Infectious Diseases and Therapy. Vol. 6, No. 2, 2021, pp. 50-60. doi: 10.11648/j.ijidt.20210602.12

Received: April 7, 2021; Accepted: April 22, 2021; Published: May 14, 2021

\begin{abstract}
The SARS-CoV-2 disease (COVID-19) is an infection caused by a virus that was discovered in the Chinese ancient city of Wuhan in December, 2019 in patients reported with pneumonia, shortness of breath and fever. Common symptoms of the virus include dry cough, nasal congestion, sore throat, loss of taste and smell, tiredness, and fever etc. Coronaviruses are single RNA-enveloped positive-sense viruses of $\sim 30 \mathrm{~kb}$. They are classified into different genera based on their genomic structure as $\alpha$, $\beta, \gamma$, and $\delta$ genera. Only the $\alpha$ and $\beta$ genera of coronavirus can be transmitted to mammals. The recent SARS-CoV-2 is associated with the $\beta$ family of coronavirus. The mode of transmission is basically via droplets from the nose or mouth of an infected person. The virus has a life cycle comprises of 5 steps; cellular attachment, invasion, replication, viral maturation and cellular escape. The mode of cellular invasion is via binding of the spike protein to angiotensin converting enzymes 2 receptor (ACE2). Knowledge on the SARS-CoV-2 RNA dependent RNA polymerase (RdRp) and spike protein, in conjunction with immunological alterations are the most promising targets for vaccine development. Manifestation of SARS-CoV-2 symptoms is very vital to the understanding of the disease. Since the inception of the virus, researchers have tried to accumulates the mild and severe symptoms of the disease in different pathological condition. The recent sharp increase in the infection rate based on the number of confirmed cases in some part of the world is worrisome, thus it has been perceived as the second wave of the virus. The second wave might last longer than expected, therefore, it is imperative to enlighten Nigerians on the immunological alteration of this virus, alongside the clinical manifestations that can arise due to SARS-CoV-2 infection - from infants to elderly; from asymptomatic patients, mild to severe cases and in patients with preexisting medical condition. Likewise, public awareness of the different strains of SARS-CoV-19 is lacking. There is little or no comprehensive research on the different variants of the coronavirus and their impending impact on the global community.
\end{abstract}

Keywords: SASR-CoV-2, Coronavirus, Variant, Pathogenesis, Disease, Second Wave

\section{Introduction}

The discoveries of different variants of the original SARS-CoV-2 virus have become a major public health problem. Since early 2020, different variants of the virus have been reported. It is a known fact that viruses constantly mutate and coronavirus is not an exception, although not all mutation result to a significance increase in viral transmissibility and cellular evasion. From December 2020 to March, 2021, many countries, including Nigeria have experience an enormous increase in the number of SARS-CoV-2 active cases. Public awareness of the different strains of SARS-CoV-19 is lacking. There is little or no comprehensive research on the different variants of the coronavirus and their impending impact on the 
global community. This increase in infection rate has been attributed to the emerging of new mutations from the original strain of the virus, therefore, ushering the world into the "Second wave" of the pandemic. Two of the major countries affected by the second wave of the virus are the United Kingdom and South Africa. In late 2020, the United Kingdom discover a new variant of coronavirus with mutation on the viral genome, especially in the spike glycoprotein called 501Y VI (from the B. 1.1.7 family). As of late December 2020, the United Kingdom recorded more than 3000 cases of the new variant. Sequent to the UK variant another variant was discovered in South Africa in October 2020 that has mutations different from that of the UK variant called the 501Y. V2. These new variants have been declared variants of concerned as the risk associated with them is said to be very high, with high impact on health care systems.

In late December, a new variant was correspondingly discovered in a sample collected in Osun State, Nigeria. This variant is called the B. 1.525 variant with an E. 4.8.4 mutation. The variant is said to have a different lineage from the other two variants discovered in UK and South Africa. Recently Nigeria has recorded a high number of COVID-19 cases, which has stimulated a public health concern and implementation of stringent measures to adopt the non-pharmaceutical preventive strategies.

The first wave of the coronavirus- 2 stated in the Chinese ancient city of China from patients reported to be suffering from pneumonia and other respiratory related infection. As of January, 2020, the Chinese Government confirmed the virus as novel type of coronavirus, later named severe acute respiratory syndrome-coronavirus - 2 (SARS-CoV-2) or coronavirus-19 (COVID-19) to differentiate it from the former SARS-CoV that was discovered in 2002. Since then, the global infection rate has been on the increase, rising public health concern about the virus. On the $11^{\text {th }}$ of March, 2020, the novel virus was declared a pandemic by the World Health Organization (WHO) due to the high increase number of infections. Renominations and different measures were taking to curb the overwhelming health challenges. SARS-CoV-2 have cause devastating impact globally on human lives, it has affected human activities with much impact on the global economy. The main viral clinical manifestation of the includes shortness of breath, sore throat, dry cough, nasal congestion, fever, muscle weakness, chest tightness, excessive mucus production, pneumonia, and acute respiratory distress syndrome [1-7]. Among these manifestation, fever, dry cough and body weakness are regarded as the most prevalent symptoms in SARS-CoV-2 patients [8, 9].

Viral genetic sequencing from five hospitalized patient with pneumonia between mid to late December, 2019 has shown similarities with the previous $\beta$ coronavirus $(\beta-\mathrm{CoV})$ strain in all the patients [10]. After the genetic sequence of the $\beta-\mathrm{CoV}$, it was discovered to be $88 \%$ identical to the earlier genetic sequence of two SARS-like coronaviruses: batSL-CoVZC45 and bat-SL-CoVZXC21, all from bat. It was also discovered to be $50 \%$ identical to the genetic sequence of the Middle East Respiratory Syndrome coronavirus (MERS-CoV) that was discovered in 2012 [11]. These similarities have served as a gate way to understanding the structure and molecular pathogenesis of the novel coronavirus. Furthermore, investigation on the virus by Chinese scientist has reveal that the viral invasion requires angiotensin-converting enzymes 2 (ACE2) receptor during cellular attachment to the host cell.

While vaccines are been develop by scientist all over the world, we cannot shy away from the fact that more strains of the virus might surface. Nobody knows the virulency that will accompany some of these mutated future variants. That said, public understanding of SARS-CoV-2 immunological alteration, clinical manifestation and latest variants are very important. This paper aims to expatiate on the pathogenesis of SARS-CoV-2, the immunological mechanism of cellular invasion, clinical manifestation in different disease conditions, and latest variants. This is to enlighten Nigerians on the latest development on the current mutation arising from the SARS-CoV-2 virus.

\section{Biochemical Fundamentals of SARS-CoV-2 Infection}

\subsection{SARS-CoV-2 Invasion and Replication}

The advent of the Severe Acute Respiratory Syndrome Corona Viruses -2 (SARS-CoV-2) in late 2019 is said to have destabilize health system across the world. This comes 16 years after the first coronavirus- 1 surfaced in 2002 and 8 years after the MERS-CoV in 2012. A sound knowledge on the SARS-CoV virus, plus the MERS-CoV virus have led to the detailed description of the SARS-CoV-2 pathogenesis. Although, the pathogenesis of SARS-CoV-2 derived from previous pathogenic mechanism of severe acute respiratory syndrome (SARS) and also that of the Middle East Respiratory Syndrome (MERS) have been reported in recent papers. Research regarding the pathophysiology linked to the SARS-CoV-2 pathogenesis will provide additional insight, shading light on the overall infection mechanism of SARS-CoV-2, replication and manifestation on the host organism. The Severe Acute Respiratory Syndrome - Corona Viruses - 2 (SARS-CoV-2) are positive-sense single RNA-enveloped strand of about $\sim 30 \mathrm{~kb}$ [12]. They are categorized into $\alpha, \beta, \gamma$, and $\delta$ genera based on the structure of their genome [13]. Only the $\alpha$ and $\beta$ genera of coronavirus can be transmitted to mammals. The recent SARS-CoV-2 is associated with the $\beta$ family of coronavirus. The mode of transmission is basically via droplets from the nose or mouth [12] of an infected person. The virus has a life cycle comprises of 5 steps; cellular attachment, penetration, biosynthesis, viral maturation and release [12]. Upon infection, the virus attaches itself to the mucosa layer of the respiratory track via a functional host receptor called angiotensin-converting enzymes - 2 (ACE2) through its spike protein via proteolytic cleavage [14]. This protein consists of two subunits (S1 and the S2 subunit) [15]. Penetration of the virus occur through endocytosis and membrane fusion [20]. The viral spike protein binds to the cell surface ACE2 of the host tissue 
thereby mediating fusion between the host cell membrane and the viral membrane, with subsequent cleavage by a host furin-like protease [16]. Once the virus gets into the cell, the viral genome begins the replication process in the nucleus of the cell, the open reading framela (ORF1a) and open reading frame 1ab (ORF1ab) are translated to synthesize the polyproteins 1a (PP1a) and polyprotein 1ab (PP1ab). The ORF1a encrypts the enzyme Protease which cleave to PP1a and PP1ab to synthesize non-structural proteins (nsps). The nsps $1 \mathrm{a}$ and $1 \mathrm{ab}$ the form the RNA replicase transcription complex. The transcription complex is responsible in the formation of a negative sense strand [17]. Fragmentation of the negative sense strand via discontinuous transcription occurs leading to the synthesis of the coronavirus structural proteins; Spike (S), membrane (M), envelop (E) and nucleocapsid $(\mathrm{N})$ in the endoplasmic reticulum [8]. The structural viral messengers are then translated to proteins via the rough endoplasmic reticulum and passed down to the Golgi apparatus through the Endoplasmic Reticulum - Golgi intermediate compartment (ERGIC) for budding and assembly (maturation) and later release through exocytosis [15]. Cryogenic electron microscopy (CryoEM) of the virus have reveal a $3 \mathrm{D}$ structure of the receptor-binding domain (RBD) located in S1 protein subunit. Structural characterization of the virus has confirmed a similar RBD mechanism of action with earlier SARS-CoV and MERS-CoV [15]. Although, structural difference was reported between the RBD of SARS-CoV and SARS-CoV-2. While RBD of SARS-CoV is tightly bound to the N-side domain of the adjoining protomer in the down conformation, the RBD of SARS-CoV-2 is closely inclined towards the central site in the down conformation [11]. Interestedly, the binding dissociation constant (KD) of SAR-CoV-2 Spike (S) proteins to ACE2 is $14.7 \mathrm{~nm}$, while that of SARS-CoV Spike proteins is $325.8 \mathrm{~nm}$ [11]. This explained the high affinity of SARS-CoV-2 S proteins to ACE2 than SARS-CoV S proteins [15]. Unsimilar to SARS-CoV-2 which is mediated by polybasic cleavage at $\mathrm{S} 1 / \mathrm{S} 2$ by cellular cathepsis $\mathrm{L}$ and the transmembrane protease serine 2 (TMPRSS2) [1-3], MERV-CoV binds to the dipeptidylpeptidase-4 (DPP4) receptor followed by membrane fusion and viral invasion [15]. Similarly, to SARS-CoV, MERV-CoV is mediated by the furin-activation invasion [18], while SARS-CoV-2 is facilitated by clathrin-dependent and -independent endocytosis [19, 20].

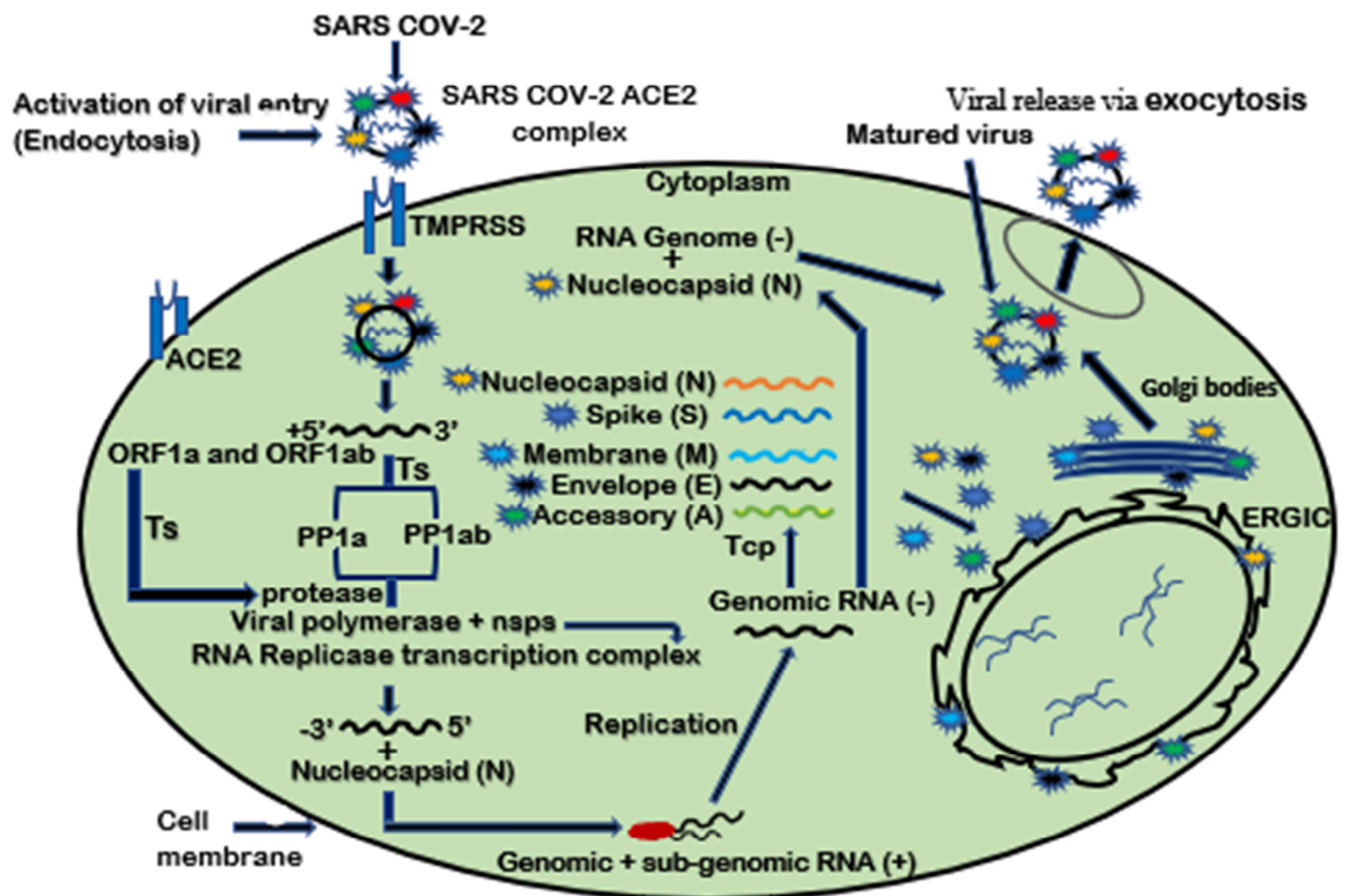

Figure 1. Pathogenesis and replication mechanism of SARS-CoV-2 in human host cell. Viral inversion, replication, transcription (Tcp), translation (TS), maturation and release.

\subsection{Immunological Alteration of SARS-CoV-2 Infection}

Immunological modification accompanying SARS-CoV-2 infection largely depends on the innate and adaptive immune response of the host organism, initiating and mounting strong immune response to the invading pathogen. This immunological response aid in controlling further infection, reduce organ injuries and facilitate speedy recovery of already 
damage tissues. Here, we elucidate hypothetically the vital human immunological responses accompanying SARS-CoV-2 infection based on previous understanding of the immune response induced by $\mathrm{CoV}$.

SARS-CoV infection is accompanied by host organism immunological response, some of these responses can help dictate the present of the virus in the host system. In newly infected SARS-CoV-2 patients, the immune system of the host organism generates specific IgM antibodies within few days after viral infection [21-23].

Manifestation of SARS-CoV-2, alongside MERS-CoV and SARS-CoV infection is known as Acute respiratory distress syndrome (ARD) [15]. The mechanism of ARD is said to be inter-linked with the response of the innate immune system to the SARS-CoV-2 viral infection. SARS-CoV-2 invasion into the cell trigger the immune system to initiate the process of inflammatory response and activation of massive production of cytokine this mechanism is called cytokines syndrome. Cytokine syndrome is the over production of deadly pro-inflammatory cytokines such as IFg1, IFN-a, IL-1b, IL6, IL-12, IL-18, IL-33, TNF-a etc. [24, 25]. These chemokines and cytokines in a great capacity, damage affected organ tissues leading to organ failure in severe conditions of SARS-CoV-2 infections [15].

Survival strategies adopted by SARS-CoV and MERS-CoV during viral invasion believed to be employed by SASR-CoV-2, is the development of double membrane shield, lacking pathogen associated recognition pattern. This prevents pattern recognition receptor to tag the virus for immediate immune action. The above process allows for viral genomic replication without immune response [26]. However, type 1 interferon - IFN (IFN-a and IFN-b) have been reported to elicit immune response upon SARS-CoV and MERS-CoV infection, but it is said to be inhibited upon expression by melanoma differentiated association proteins gene 5 (MDA5), followed by downregulation of major histocompatibility complex (MHC) class 1 and II molecules and lower activation of T-cells lymphocytes. [27-31].

\section{Expected Clinical Symptoms in SARS-CoV-2 Patients}

\subsection{General Clinical Manifestation}

Manifestation of SARS-CoV-2 symptoms is very vital in understanding the disease. Since the inception of the virus in December, 2019, researchers have tried to accumulates the mild and severe symptoms of the disease. The recent continue increase in the infection rate (based on the number of confirmed cases) due to the second wave of the virus, especially here in Nigeria is worrisome, therefore, there is need to enlighten people on the various clinical manifestation that can arise due to SARS-CoV-2 infection - from infants to elderly; from asymptomatic persons, mild patients to even severe cases. The most common and distinctive clinical manifestation include shortness of breath, sore throat, dry cough, nasal congestion, fever, muscle weakness, chest tightness, excessive mucus production, pneumonia, and acute respiratory distress syndrome [4-8]. Among these manifestation, fever, dry cough and body weakness are regarded as the most prevalent symptoms in SARS-CoV-2 patients $[9,10]$. Noteworthy, approximately $90 \%$ percent of SARS-CoV-2 patients come up with more than one symptom $[32,33]$. Notwithstanding, some patients might also experience unlikable or additional symptoms to the above as clinical manifestation may differ between people with pre-existing health challenges. In severe condition, patients might present respiratory, neurological, olfactory, gastrointestinal, ophthalmic, dermatological, cardiac, and rheumatological manifestation [34]. Baj et al. also reported that among $81 \%$ of death investigated from Wuhan, $41.91 \%$ of infected patients die due to respiratory failure, $19.75 \%$ due to septic shock, $16.05 \%$ due to multiple organ failure and $8.64 \%$ due to cardiac arrest [34]. Pathological changes accompanying SARS-CoV-2 infection are becoming progressively apparent. Consequently, a review on the comprehensive clinical manifestation of SARS-CoV-2 disease in different stage of development and in patients with some selected preexisting health challenges is of optimum importance. This will be useful in generating advance clinical management systems in the management of SARS-CoV-19 patients in the different groups of the general population.

\subsection{SARS-CoV-2 in Pregnant Women}

The manifestation of SARS-CoV-2 in pregnant women has been reported to be associated with Gastrointestinal symptoms alongside the general symptoms (fatigue, myalgia, fever, cough, dyspnea or difficulty in breathing). These symptoms include diarrhea, abdominal pain, nausea, loss of appetite and abdominal distension [35]. A study has also reported $10.6 \%$ of patients with SARS CoV and 30\% of patients with MERS coronavirus experience diarrhea [36]. Further investigation from Chan et al. revealed that MERS can survive in the gastrointestinal tract, thereby causing intestinal infection [36]. Notwithstanding, SARS-CoV-2 also cause disorder of the intestinal flora and can result to gastrointestinal manifestation [37]. SARS-CoV-2 have also been detected in the stool of COVID-19 patients [38, 39]. These evidences suggest a possibility of SARS-CoV-2 invasion and transmission via oral-fecal, mother-fetal, and breast-milk transmission of SARS-CoV-2 indicating the urgent need for effective diagnostic models. Therefore, the manifestation of gastrointestinal symptoms with or without the general respiratory symptoms might indicate a sign of the viral infection, especially in pregnant women.

\subsection{SARS-CoV-2 and Patients with Pre-existing Cardiac Diseases}

The increase rising cases of SARS-CoV-2 following the second wave has raise serious concern, especially with people with pre-existing medical condition. Here, we shade more light on the clinical manifestation of SARS-CoV-2 in patients with pre-existing cardiovascular disease. Cardiac diseases patients, 
specifically hypertension and coronary heart disease are at greater risk of developing severe and fetal COVID-19 [40]. Angiotensin converting enzymes (ACE) inhibitors and its receptors blockers (ARB) have been used in the management of SARS-CoV-2 [40]. ACE-inhibitors or receptor blockers are also used as anti-hypertensive drugs. These drugs are used as first line therapy in hypertensive patients to slow down the progression of renal dysfunction. The mechanism of action of these drugs is in decreasing the formation of ACE-2 leading to systemic dilation of the arteries and veins, with a subsequent decrease in arterial blood pressure. Li et al. reported a meta-analysis of six groups with 1527 patients presenting hypertension as a co-morbidity in 17.1 and $16.4 \%$ of the patients [41]. In addition, Zhou et al. recorded $86 \%$ of death from patients with COVID-19 with $45 \%$ due to arterial hypertension, indicating the highest percentage rate of death in their entire work [42]. According to a retrospective study by Gao et al. of 2877 hospitalized patients, $29.5 \%$ were reported to have had hypertension earlier on before the studies. Therefore, pre-existing cardiovascular condition in SARS-CoV-2 patients can be linked with an increased risk of death [43].

Management of COVID-19 patients with a prior cardiovascular disease is controversial, this is due to the used of ACE-2 inhibitors drugs and ARB were earlier believed to induced higher viral loads [44]. However, the assumption has been descanted due to the understanding of the ACE-2 expression mechanism [43]. Cardiovascular clinical manifestation accompanying SARS-CoV-2 infection include hypoxia-induced myocyte injury, immune-mediated cytokine storm and increase in cardiac troponin $[45,46]$.

In 2003, SARS-CoV-1 was recognized as risk factor in pre-existing diabetic patients. In another similar case, the Middle East Respiratory Syndrome (MERS) was recorded to have post further threat and serious complications to diabetic patients. [47, 48], likewise, SARS-CoV-2. COVID-19 has been proved to increase the risk of complications in pre-existing diabetic patients [49]. Generally, people with diabetic history are susceptible to SARS-CoV-2 infection. This is due to the defect in their innate immune system affecting phagocytosis, neutrophil chemotaxis, and cellular-mediated immunity [50]. Secondary disease conditions such as inflammatory tissue damage, nephropathy, coagulation dysfunction, cardiovascular diseases are also predisposing risk factors associated with SARS-CoV-2 infection. Wu and McGoogan [51] reported a comprehensive epidemiological study of 72,314 patients with SARS-CoV-2 in China indicating patients with diabetes having a mortality rate three times higher than all patients with SARS-CoV-2. In severe case of diabetic infection, the key complications are usually caused by hyperactive inflammatory state and coagulative dysfunction posing high risk of thrombosis and kidney damage [52]. The homeostasis dysfunction of the coagulation system, fibrinolytic pathways and inflammation expressed in severe case of diabetic condition also manifest in severe SARS-CoV-2 infection [52]. Yan et al. reported that patients with infection had higher blood sugar levels compared with patients with different viral agent [53].
Another study in 174 COVID-19 patients in Wuhan, China reported higher concentration of inflammatory markers and incidence of coagulation leading to high death rate than non-diabetic patients [54]. The distressing relationship between SARS-CoV-2 and diabetes is assumed to be as a result of viral binding to ACE2 receptor since the same mechanism has been postulated to be the cause of hyperglycemia in earlier SARS-CoV [55]. Studies have also revealed that SARS-CoV-2 infection cause insulin tissue resistivity [32], However, the specific role of the virus in insulin resistance is not fully understood. Clinical manifestation of diabetic SARS-CoV-2 patient include but not excluded to mild swelling of the bilateral tonsils, elevated blood glucose level, decrease of oxygen saturation, increase in the number of neutrophils and lymphocytes, decrease in total serum proteins and elevated serum glycated hemoglobin [56]. However, most of these symptoms with the exception of blood glucose level and glycated hemoglobin can be express in other COVID-19 patients [57].

\subsection{SARS-CoV-2 and Patients with Kidney Disease}

SARS-CoV-2 and Kidney disease have been reported in very few papers since the outbreak of the novel virus. However, patients with preexisting kidney disease, especially those with kidney transplant [58] are most at risk of developing complications from COVID-19 infection due to immunosuppression [59]. Although very little is known about the effect of SARS-CoV-2 on immunocompromised patients, especially those with kidney related disease or have undergone a kidney transplants, complications arising include hypertension, diabetes mellitus, cardiovascular disease and Bilateral Pneumonia. A study conducted in Wuhan by Wu et al., on hospitalized patients recorded a severe disease condition for patients with pre-existing kidney disease compare to the general study population [60]. However, the findings of $\mathrm{Wu}$ et al was not typical to the general clinical presentation of COVID-19 patients as they recorded lack of fever and cough in patients on dialysis. This was also observed by a Xiong et al. in a previous study [61]. Notwithstanding, symptoms presented were fatigue (in 59\%) and anorexia (in $57 \%$ ) [60]. Therefore, there is great need for clinical nephrologist to fully be aware of the best evidence associated with SARS-CoV-2 infection and pre-existing clinical challenges with higher mortality rate. These will be useful in generating an advance clinical management system in the management of SARS-CoV-19 patients with pre-existing kidney challenges.

\subsection{SARS-CoV-2 Complication in Cancer Patients}

Cancer patients are among the highly vulnerable subclass of the general population in SAR-CoV-2 crisis. This might be due to increasing risk of immunosuppressive chemotherapy treatment, resulting to higher severity of the diseases [62]. Frequent hospital visitation by cancer patients has also created opportunity for viral infection, especially COVID-2. Noteworthy, the SARS-CoV-2 mortality rate $5.6 \%$ in cancer 
patients is twice higher than $2.3 \%$ recorded in the general population [63]. These conditions can result in death as describe by Minotti et al. [64]. In addition, SARS-CoV-19 patients with pre-existing cancer condition are also vulnerable to other SARS-CoV-2 related complications. The disruption of the continuous medical care provided to cancer patients, including diagnostic and therapeutic management such as magnetic resonance imaging, mammograms, biopsies, position emission tomography scans, elective surgeries, chemotherapy, and radiotherapy has been seriously affected by SARS-CoV-2, hence, hindering diagnosis, management and treatment of cancer patients $[64,65]$. A study reported by Seth et al. of two lung cancer patients with a medical history of diabetes and hypertension before SARS-CoV-2 diagnosis shows that they were initially asymptomatic with clear bilateral respiratory auscultation [62, 65]. Nevertheless, after the patients undergo unilateral pneumonectomy, the patients experienced chest tightness, dyspnea, and decreased oxygen saturation [62]. Study conducted by Hrusak et al. on 9 children with cancer reported that none of the children required an ICU admission, hence stated that children undertaking cancer treatment experienced milder disease outcomes and should therefore proceed with their cancer treatment $[63,66]$. Cell proliferation in breast and lung cancer has been reported to be associated with proangiogenic factors, which is as a result of the conversion of angiotensin I to angiotensin II by ACE [67, 68]. In SARS-CoV-2 viral entry, the virus inhibits the ACE2 binding to it receptor, thereby reducing the amount of active ACE2 and subsequently, resulting to antiproliferation, anti-inflammatory effects and promotion of tumorigenesis [69-72]. SARS-CoV-2 may intensify angiogenesis and cancer progression, therefore, there is need for more research on the effect of SARS-CoV-2 in cancer patients and possible health management strategy to curb the adverse effects caused by the virus, especially the administration of immunosuppressant therapy to cancer patients. Severe case of SARS-CoV-2 manifestation include but not exclusively acute respiratory distress syndrome, Septic shock, acute myocardial infarction [73].

\section{SARS-CoV-2 Second Wave}

Following the intensive research from scientist around the globe, several highly mutated and infectious SARS-CoV-2 variants have been discovered since November, 2020. Insight into these variants' evolution is necessary for upgrading the early PCR diagnostic test of the virus. However, viruses are known for their common ability to mutate and SARS-CoV-2 is not an exception. An article from nature reported that the mutation of SARS-CoV-2 occur at a rate of about 1-2 mutation per month [74]. Notwithstanding, the SARS-CoV-2 has been discovered to mutate significantly in shorter duration of time [75]. Scientist have speculated that there will be more variants of the SARS-CoV-2 in the upcoming months, as rapid uncontrolled transmission of the virus is taking place in many parts of the world, especially in Africa. The World Health Organization (WHO) in $28^{\text {th }}$ January stated that African has recorded over 175000 new SARS-CoV-19 cases and more than 6200 deaths in the last few weeks with about $50 \%$ increase in infection rate between late December and late January, 2021. Furthermore, death rate has double within this period with most occurrences in the Southern and Northern African countries. These significant outbursts might be related to the 501Y. V2 SARS-CoV-2 variant that was identified in South Africa in October, 2020 with a mutation at the receptor binding domain (RBD) of the virus, belonging to the B. 1.351 lineage. This COVID-19 variant has been detected in other African countries such as Nigeria, Ghana, Gambia and also in some part of UK. Alongside the Southern African variant, the first SARS-CoV-19 variant to be dictated is the UK variant (501Y. VI). The UK variant has been discovered to have a lot of mutations in the virus genome, especially in the spike glycoprotein, belonging to the B. 1.1.7 lineage [58]. It has also shown higher transmission rate and disease severity [76]. In late December, a new variant was correspondingly discovered in a sample collected in Osun State, Nigeria. This variant is called the B. 1.525 variant with an E. 4.8.4 mutation. The variant is said to have a different lineage from the other two variants discovered in UK and South Africa. Recently Nigeria has recorded a high number of COVID-19 cases, which has stimulated a public health concern and implementation of stringent measures to adopt the non-pharmaceutical preventive strategies. The public alarm caused by the new variant is speculated to be more contagious, similar to that of the UK variant. As the second wave of the pandemic is emerging, new variants have been detected in Portugal amidst the pandemic. However, study on the Portugal variants has suggested a more contagious strain than the original strain of the virus but less deadly. Investigations on the SARS-CoV-2 latest variants are still ongoing to ascertain the virulent impact of the new strains. Recently, $25^{\text {th }}$ January, 2021, Minnesota recorded it first case of SARS-CoV-2 virus caused by a different variant from the already existing strains. The variant is named Brazil P. 1 variant, also said to be more contagious than the original COVID-19 strain, as more investigations are going on, the severity of the Brazil P. 1 strain is yet to be ascertain. Finding on the SARS-CoV-2 viral evolution has suggest a rapid and active global epidemiological surveillance system to quickly ascertain the level of viral transmission, virulency and death rate, as a matter of urgency in proactively managing the pandemic. 


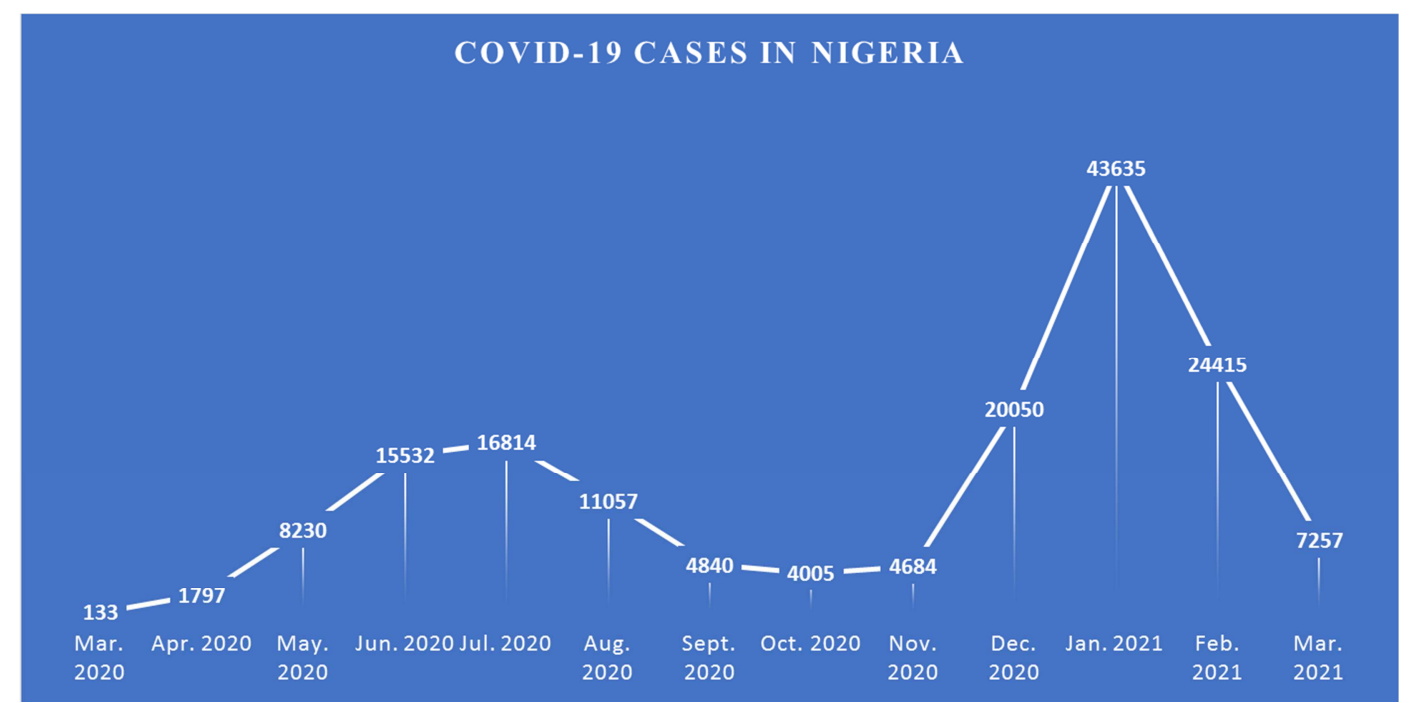

Figure 2. SASR-CoV-2 cases in Nigeria from March, 2020 to March 2021. Nigeria second wave: Nov. 2020-Apr. 2021. Highest number of infected cases were recorded in Jan. 2021, signifying the peak of the SARS-CoV-2 second wave.

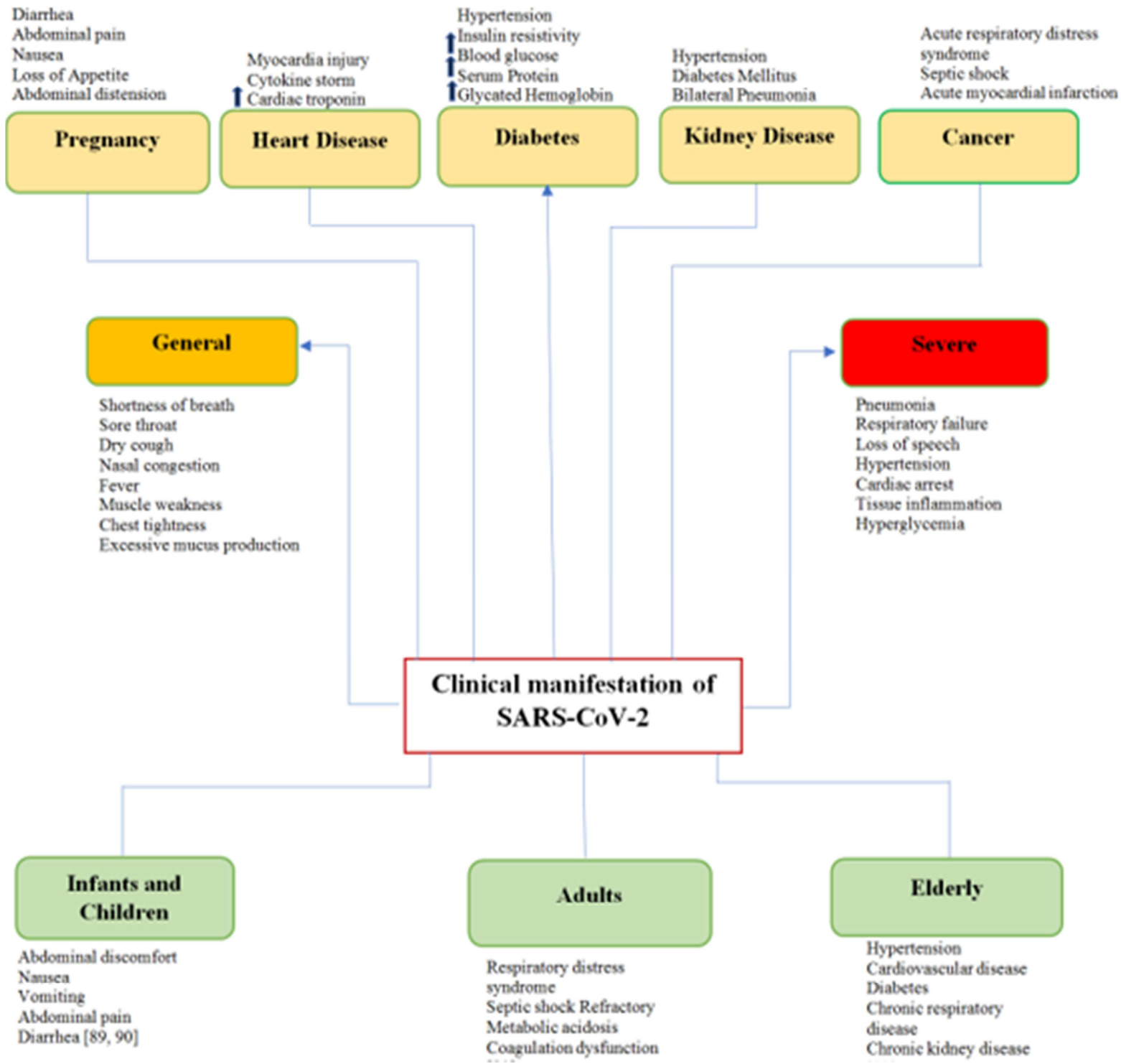

Figure 3. Clinical manifestation of SARS-CoV-2 infection on human host [77-80]. 


\section{Conclusion}

Viruses constantly mutate and coronavirus is not an exception, while most mutations might not have significant impact on the virulency of the virus, some mutations might lead to increase in viral transmissibility and cellular evasion. Understanding the molecular basic of viral infection is very imperative in drug design against emerging variants. It is also essential for the health workers to be fully aware of the clinical manifestation of the viral infection for proper management of the disease. Nigerian government are also saddled with the responsibility of enlightening the general public about the dangers associated with viral mutation and the importance of strict adhering to non-pharmaceutical interventions in order to manage viral infection and mutation.

\section{Future Recommendation}

As the second wave of the pandemic is claiming more lives all over the world, it is highly essential to keep track of emerging viral strains through effective surveillance system sample collection and regional sequencing. Using structural Biological modelling, viral mutation should be predicted to proactively be incorporated in future vaccine designs to combat new variants of global concern before they even arrive.

\section{Conflict of Interest Statement}

The authors declare that they have no competing interests.

\section{Reference}

[1] Lei, S., Jiang, F., Su, W., Chen, C., Chen, J., Mei, W., Zhan, L. Y., Jia, Y., Zhang, L., Liu, D., et al. (2020). Clinical Characteristics and Outcomes of Patients Undergoing Surgeries During the Incubation Period of COVID-19 Infection. EClinical Medicine, 21, 100331.

[2] Guan, W. J., Ni, Z. Y., Hu, Y., Liang, W. H., Ou, C. Q., He, J. X., Liu, L., Shan, H., Lei, C. L., Hui, D. S. et al. (2019) Clinical Characteristics of Coronavirus Disease in China. N. Engl. J. Med., 382 (18), 1708-1720.

[3] Xie, J., Tong, Z., Guan, X., Du, B., \& Qiu, H. (2020) Clinical Characteristics of Patients Who Died of Coronavirus Disease 2019 in China. JAMA Netw., 3, e205619.

[4] Wang, Z., Chen, X., Lu, Y., Chen, F., \& Zhang, W. (2020) Clinical Characteristics and Therapeutic Procedure for four Cases with 2019 Novel Coronavirus Pneumonia Receiving Combined Chinese and Western medicine Treatment. Biosci. Trend, 14 (1), 64-68.

[5] Kim, E. S., Chin, B. S., Kang, C. K., Kim, N. J., Kang, Y. M., Choi, J. P., Oh, D. H., Kim, J. H., Koh, B., Kim, S. E., et al. (2020) Clinical Course and Outcomes of Patients with Severe Acute Respiratory Syndrome Coronavirus 2 Infection: A Preliminary Report of the First 28 Patients from the Korean Cohort Study on COVID-19. J. Korean Med. Sci., 35 (13), e142.

[6] Tu, H., Tu, S., Gao, S., Shao, A., \& Sheng, J. (2020) Current
Epidemiological and Clinical Features of COVID-19; A Global Perspective from China. J. Infect., 81 (1): 1-9.

[7] Fu, L., Wang, B., Yuan, T., Chen, X., Ao, Y., Fitzpatrick, T., Li, P., Zhou, Y., Lin, Y. F., Duan, Q., et al. (2020) Clinical Characteristics of Coronavirus Disease 2019 (COVID-19) in China: A systematic Review and Meta-analysis. J. Infect., 80 (6), 656-665.

[8] Sun, P., Qie, S., Liu, Z., Ren, J., Li, K., \& Xi, J. (2020) Clinical Characteristics of Hospitalized Patients with SARS- CoV-2 Infection: A single arm Meta-analysis. J. Med. Virol., 92, 612617.

[9] Shi, F., Yu, Q., Huang, W., \& Tan, C. (2020) 2019 Novel Coronavirus (COVID-19) Pneumonia with Hemoptysis as the Initial Symptom: CT and Clinical Features, Korean J. Radiol., $21(5), 537-540$.

[10] Bosch, B. J., van der Zee, R., de Haan, C. A., \& Rottier, P. J. (2003) The coronavirus spike protein is a class I virus fusion protein: structural and functional characterization of the fusion core complex. J Virol, 77 (16), 8801-11.

[11] Wrapp, D., Wang, N., Corbett, K. S., Goldsmith, J. A., Hsieh, C. L., Abiona, O., Graham, B. S., \& McLellan, J. S. (2020) Cryo-EM Structure of the 2019-nCoV Spike in the Prefusion Conformation, Science, 367 (6483), 1260-1263.

[12] Yuki, K., Fujiogi, M., \& Koutsogiannaki, S. (2020) COVID-19 Pathophysiology: A Review. Clinical immunology, 215, 108427.

[13] Rabi, F. A., Al Zoubi, M. S., Kasasbeh, G. A., Salameh, D. M., \& Al-Nasser. A. D. (2020) SARS-CoV-2 and Coronavirus Disease 2019: what we know so far. Pathogens., 9 (3), 231.

[14] Li, W., Moore, M. J., Vasilieva, N., Sui, J., Wong, S. K., Berne, M. A., Somasundaran, M., Sullivan, J. L., Luzuriaga, K., Greenough T. C., Choe, H., \& Farzan, M. (2003) Angiotensin-Converting Enzyme 2 is a Functional Receptor for the SARS coronavirus., Nature., 426, 450-454.

[15] Muhammed, Y. (2020). Molecular Targets for COVID-19 Drug Development: Enlightening Nigerians about the Pandemic and Future Treatment. Biosaf Health., 2 (4), 210-216.

[16] Gui, M., Song, W., Zhou, H., Xu, J., Chen, S., Xiang, Y., \& Wang, X. (2017) Cryo-electron Microscopy Structures of the SARS-CoV Spike Glycoprotein Reveal a Prerequisite Conformational State for Receptor Binding, Cell Research, 27 (1), 119-129.

[17] Cevik, M., Kuppalli, K., Kindrachuk, J., \& Peiris, M. (2020) "Virology, Transmission, and Pathogenesis of SARS-CoV-2," BMJ, 371, 1-6.

[18] Mille, J. K., \& Whittaker, G. R. (2014) Host Cell Entry of Middle East Respiratory Syndrome Coronavirus After Two-Step, Furin-Mediated Activation of the Spike Protein, Proc. Natl. Acad. Sci. U. S. A., 11 (42), 15214-15219.

[19] Wang, H., Yang, P., Liu, K., Guo, F., Zhang, Y., Zhang G., \& Jiang, C. (2008) SARS Coronavirus Entry into Host Cells Through a Novel Clathrin- and Caveolae-Independent Endocytic Pathway, Cell Res., 18 (2), 290-301.

[20] Kuba, K., Imai, Y., Ohto-Nakanishi, T., \& Penninger, J. M. (2010) Trilogy of ACE2: A Peptidase in the Renin-Angiotensin System, A SARS Receptor, and A Partner for Amino Acid Transporters, Pharmacol. Ther., 128 (1), 119-128. 
[21] Yang, J., Zheng, Y., Gou, X., Pu, K., Chen, Z., Guo, Q., Ji, R., Wang, H., Wang, Y., \& Zhou, Y. (2020) Prevalence of Comorbidities and its Effects in Patients Infected with SARS-CoV-2: A Systematic Review and Meta-analysis. Int. J. Infect. Dis., 94, 91-95.

[22] Lu, R., Zhao, X., Li, J. Niu, P. Yang, B. Wu, H. Wang, W., Song, H., Huang, B., Zhu, N. et al. (2020) Genomic Characterisation and Epidemiology of 2019 Novel Coronavirus: Implications for Virus Origins and Receptor Binding. Lancet, 395 (10224) 565-574.

[23] Tai, W., He, L., Zhang, X., Pu, J., Voronin, D., Jiang, S., Zhou, Y., \& Du, L. (2020) Characterization of the Receptor-Binding Domain (RBD) of 2019 Novel Coronavirus: Implication for Development of RBD Protein as a Viral Attachment Inhibitor and Vaccine. Cell, Mol. Immunol., 17 (6), 613-620.

[24] Xu, Z., Shi, L., Wang, Y., Zhang, J., Huang, L., Zhang, C., Liu, S., Zhao, P., Liu, H., Zhu, L., Tai, Y., Bai, C., Gao. T, Song, J., Xia, P., Dong, J., Zhao, J., \& Wang, F. S., (2020) Pathological Findings of COVID-19 Associated with Acute Respiratory Distress Syndrome, Lancet Respir. Med., 8 (4) 420-422.

[25] Huang, C., Wang, Y., Li, X., Ren, L, Zhao, J., Hu, Y, Zhang, L., Fan, G., Xu, J., Gu, X., Cheng, Z., Yu, T., Xia, J., Wei, Y., Wu, W., Xie, X., Yin, W., Li, H., Liu, M., Xiao, Y., Gao, H., Guo, L., Xie, J., Wang, G., Jiang, R., Gao, Z., Jin, Q., Wang, J., \& Cao, B. (2020) Clinical Features of Patients Infected with 2019 Novel Coronavirus in Wuhan, China, Lancet, 395 (10223), 497-506.

[26] Williams, A. E., \& Chambers, R. C. (2014) The Mercurial Nature of Neutrophils: Still an Enigma in ARDS? Am. J. Phys. Lung Cell. Mol. Phys., 306 (3), L217-230.

[27] Snijder, E. J., van der Meer, Y., Zevenhoven-Dobbe, J., Onderwater, J. J., van der Meulen, J., Koerten, H. K., \& Mommaas, A. M. (2006) Ultrastructure and Origin of Membrane Vesicles Associated with the Severe Acute Respiratory Syndrome Coronavirus Replication Complex, J. Virol., 80 (12), 5927-5940.

[28] Masters, P. S. (2006) The Molecular Biology of Coronaviruses. In Advances in Virus Research; Elsevier: Amsterdam, The Netherlands, 66, 193-292.

[29] Fischer, F., Stegen, C. F., Masters, P. S., \& Samsonoff, W. A. (1998) Analysis of Constructed E Gene Mutants of Mouse Hepatitis Virus Confirms a Pivotal Role for E Protein in Coronavirus Assembly., J. Virol., 72 (10), 7885-7894.

[30] Chu, H., Chan, J. F.-W., Yuen, T. T.-T., Shuai, H., Yuan, S., Wang, Y., Hu, B., Yip, C. C.-Y., Tsang, J. O.-L., Huang, X. et al. (2020) Comparative Tropism, Replication Kinetics, and Cell Damage Profiling of SARS-CoV-2 and SARS-CoV with Implications for Clinical Manifestations, Transmissibility, and Laboratory Studies of COVID-19: An observational Study. Lancet Microbe, 1 (1), e14-e23.

[31] Lebeau, G., Vagner, D., Frumence, É., Ah-Pine, F., Guillot, X., Nobécourt, E., Raffray, L., \& Gasque, P. (2020) Deciphering SARS-CoV-2 Virologic and Immunologic Features. International journal of molecular sciences, 21 (16), 5932.

[32] Wang, R., Pan, M., Zhang, X., Han, M., Fan, X., Zhao, F., Miao, M., Xu, J., Guan, M., Deng, X. et al. (2020) Epidemiological and Clinical Features of 125 Hospitalized Patients with COVID-19 in Fuyang, Anhui, China, Int. J. Infect. Dis., 95, 421-428.
[33] Yan, Y., Shin, W., Pang, Y., Meng, Y., Lai, J., You, C., Zhao, H., Lester, E., Wu, T., \& Pang, C. H. (2020) The First 75 Days of Novel Coronavirus (SARS-CoV-2) Outbreak: Recent Advances, Prevention, and Treatment, Int. J. Environ. Res. Public Health, 17 (7), 2323.

[34] Jacek, B., Hanna, K.-J., Grzegorz, T., Grzegorz, B., Marzanna, C., Elżbieta, S., Alicja, F., Kaja, K., Wojciech, F., Piero, P., \& Ryszard, M. (2020) COVID-19: Specific and Non-Specific Clinical Manifestations and Symptoms: The Current State of Knowledge. Journal of Clinical Medicine., 9 (6), 1753.

[35] Makvandi, S., Ashtari, S, Vahedian-Azimi A. (2020) Manifestations of COVID-19 in pregnant Women with Focus on Gastrointestinal Symptoms: A Systematic Review, Gastroenterol Hepatol Bed Bench., 13 (4), 305-312.

[36] Wang, D., Hu, B., Hu, C., Zhu, F., Liu, X., Zhang, J., et al. (2020) Clinical Characteristics of 138 Hospitalized Patients with 2019 Novel Coronavirus-Infected Pneumonia in Wuhan, China, JAMA, 323 (11), 1061-9.

[37] Pan, L., Mu, M., Yang, P., Sun, Y., Wang, R., Yan, J., et al. (2020) Clinical Characteristics of COVID-19 Patients with Digestive Symptoms in Hubei, China: A Descriptive, Cross-Sectional, Multicenter Study, Am J Gastroenterol, 115 (5), 766-73.

[38] Holshue, M. L., DeBolt, C., Lindquist, S., Lofy, K. H., Wiesman, J., Bruce, H., et al. (2020) First Case of 2019 Novel Coronavirus in the United States, N Engl J Med, 382, 929-36.

[39] Gu, J., Han, B., \& Wang, J. (2020) COVID-19: Gastrointestinal Manifestations and Potential Fecal-Oral Transmission, Gastroenterology, 158 (6), 1518-9.

[40] Flaherty, G. T., Hession, P., Liew, C. H. et al. (2020) COVID-19 in Adult Patients with Pre-existing Chronic Cardiac, Respiratory and Metabolic Disease: A Critical Literature Review with Clinical Recommendations, Trop Dis Travel Med Vaccines, 6, 16.

[41] Li, B., Yang, J., Zhao, F., Zhi, L., Wang, X., Liu, L., et al. (2020) Prevalence and Impact of Cardiovascular Metabolic Diseases on COVID-19 in China, Clin Res Cardiol, 109 (5), 531-8.

[42] Zhou, F., Yu, T., Du, R., Fan, G., Liu, Y., Liu, Z., et al. (2020) Clinical Course and Risk Factors for Mortality of Adult in Patients with COVID-19 in Wuhan, China: A Retrospective Cohort Study, Lancet., 395 (10229), 1054-62.

[43] Guo, J., Huang, Z., Lin, L., \& Lv, J., Coronavirus Disease 2019 (COVID-19) and Cardiovascular Disease: A Viewpoint on the Potential Influence of Angiotensin-Converting Enzyme Inhibitors/Angiotensin Receptor Blockers on Onset and Severity of Severe Acute Respiratory Syndrome Coronavirus 2 Infection, J Am Heart Assoc., 9 (7), e016219.

[44] Chu, C. M., Poon, L. L., Cheng, V. C., Chan, (3K. S., Hung, I. F., Wong, M. M. K., et al. (2004) Initial Viral Load and the Outcomes of SARS, CMAJ, 171 (11), 1349-52.

[45] Zhu, H., Rhee, J. W., Cheng, P., Waliany, S., Chang, A., Witteles, R. M., et al. (2020) Cardiovascular Complications in Patients with COVID-19: Consequences of Viral Toxicities and Host Immune Response. Curr Cardiol Rep, 22 (5), 32.

[46] Gao, L., Jiang, D., Wen, X. S., Cheng, X. C., Sun, M., He, B., et al. (2020) Prognostic Value of NT-ProBNP in Patients with Severe COVID-19, Respir Res., 21 (1), 83. 
[47] Yang, Y. M., Hsu, C. Y., Lai, C. C., Yen, M. F., Wikramaratna, P. S., Chen, H. H., et al. (2017) Impact of Comorbidity on Fatality Rate of Patients with Middle East Respiratory Syndrome, Sci Rep, 7 (1), 11307.

[48] Garbati, M. A., Fagbo, S. F., Fang, V. J., Skakni, L., Joseph, M., Wani, T. A., et al. (2016) A Comparative Study of Clinical Presentation and Risk Factors for Adverse Outcome in Patients Hospitalised with Acute Respiratory Disease due to MERS Coronavirus or other Causes. PLoS One, 11 (11), e0165978.

[49] Allard, R., Leclerc, P., Tremblay, C., \& Tannenbaum, T. N. (2010) Diabetes and the Severity of Pandemic Influenza A (H1N1) Infection, Diabetes Care, 33 (7), 1491-3.

[50] Bornstein, S. R., Rubino, F., Khunti, K., Mingrone, G., Hopkins, D., Birkenfeld, A. L., et al. (2020) Practical Recommendations for the Management of Diabetes in Patients with COVID-19. Lancet Diabetes Endocrinol, 8 (6), 546-50.

[51] Wu, Z., \& McGoogan, J. M., (2020) Characteristics of and Important Lessons from the Coronavirus Disease 2019 (COVID-19) Outbreak in China: Summary of a Report of 72 314 Cases from the Chinese Center for Disease Control and Prevention, JAMA, 323 (13), 1239-1242.

[52] Antonio, V. \& Francesco, F. (2020) The Impact of COVID-19 in Diabetic Patient, Archives of Medicine and Health Sciences, 8 (1), 167-171.

[53] Guo, W., Li, M., Dong, Y., Zhou, H., Zhang, Z., Tian, C., et al. (2020) Diabetes is a Risk Factor for the Progression and Prognosis of COVID-19, Diabetes Metab Res Rev, 3, e3319.

[54] Yang, J. K., Feng, Y., Yuan, M. Y., Yuan, S. Y., Fu, H. J., Wu, B. Y., et al. (2006) Plasma Glucose Levels and Diabetes are Independent Predictors for Mortality and Morbidity in Patients with SARS. Diabet Med, 23 (6), 623-8.

[55] Yang, J. K., Lin, S. S., Ji, X. J., \& Guo, L. M. (2010) Binding of SARS Coronavirus to its Receptor Damages Islets and Causes Acute Diabetes. Acta Diabetol, 47 (3), 193-9.

[56] Alireza, A., Milad, J., Pegah, S. \& Zeljko, V. (2020) Diabetes and COVID-19: A Systematic Review on the Current Evidences, Diabetes Research and Clinical Practice, 166. 108347.

[57] Pan, L., Mu, M., Ren, H. G., Yang, P., Sun, Y., \& Wang, R. (2020) Clinical Characteristics of COVID-19 Patients with Digestive Symptoms in Hubei, China: A Descriptive, Cross-Sectional, Multicenter Study, Am J Gastroenterol, 115 (5), 766-773

[58] Maria, A., \& Melamed, M. L. (2020) Covid-19 in Patients with Kidney Disease; CJASN, 15 (8), 1087-1089.

[59] Husain, S. A., Dube, G., Morris, H., Fernandez, H., Chang, J. H., Paget, K., Sritharan, S., Patel, S., Pawliczak, O., Boehler, M., Tsapepas, D., Crew, R. J., Cohen, D. J., \& Mohan, S. (2019) Early Outcomes of Outpatient Management of Kidney Transplant Recipients with Coronavirus Disease 2019, Clin J Am Soc Nephrol, 15 (8), 1174-1178.

[60] Wu, J., Li, J., Zhu, G., Zhang, Y., Bi, Z., Yu, Y., Huang, B., Fu, S., Tan, Y., Sun, J., \& Li, X. (2020) Clinical Features of Maintenance Hemodialysis Patients with 2019 Novel Coronavirus-Infected Pneumonia in Wuhan, China, Clin J Am Soc Nephrol, 15 (8), 1139-1145.

[61] Xiong, F., Tang, H., Liu, L., Tu, C., Tian, J. B., Lei, C. T., Liu,
J., Dong, J. W., Chen, W. L., Wang, X. H., Luo, D., Shi, M., Miao, X. P, \& Zhang, C. (2020) Clinical Characteristics of and Medical Interventions for COVID-19 in Hemodialysis Patients in Wuhan, China, J Am Soc Nephrol, 31 (7), 1387-1397.

[62] Seth, G., Sethi, S., Bhattarai, S., Saini, G., Singh, C. B., \& Aneja, R. (2020). SARS-CoV-2 Infection in Cancer Patients: Effects on Disease Outcomes and Patient Prognosis, Cancers, $12(11), 3266$

[63] Minotti, C., Tirelli, F., Barbieri, E., Giaquinto, C. \& Donà, D. (2020) How is Immunosuppressive Status Affecting Children and Adults in SARS-CoV-2 Infection? A Systematic Review, J. Infect., 81 (1), e61-e66.

[64] Serraino, D. (2020) COVID-19 and Cancer: Looking for Evidence, Eur. J. Surg. Oncol., 46 (6), 929-930.

[65] Tian, S., Xiong, Y., Liu, H., Niu, L., Guo, J., Liao, M., \& Xiao, S. Y. (2020) Pathological Study of the 2019 Novel Coronavirus Disease (COVID-19) Through Postmortem Core Biopsies., Modern Pathology, 33 (6), 1007-1014.

[66] Liang, W., Guan, W., Chen, R. Wang, W., Li, J., Xu, K., Li, C., Ai, Q., Lu, W., Liang, H., et al. (2020) Cancer Patients in SARS-CoV-2 Infection: A Nationwide Analysis in China, Lancet Oncol, 21 (3), 335-337.

[67] Vatansev, H., Kadiyoran, C., Cure, C. M., \& Cure, E. (2020) COVID-19 Infection can Cause Chemotherapy Resistance Development in Patients with Breast Cancer and Tamoxifen may Cause Susceptibility to COVID-19 Infection, Med. Hypotheses, 143, 110091.

[68] Gallagher, P. E., Cook, K., Soto-Pantoja, D., Menon, J., \& Tallant, E. A. (2006) Angiotensin Peptides and Cancer., Curr Cancer Drug Targets, 11 (4), 394-404.

[69] Xie, X., Chen, J., Wang, X., Zhang, F., \& Liu, Y. (2006) Ageand gender-related Difference of ACE2 Expression in Rat Lung. Life. Sci., 78 (19): 2166-71.

[70] Jakovac, H., (2020) COVID-19: Is the ACE2 Just a Foe? Am. J. Physiol. Lung. Cell. Mol. Physiol., 318, L1025-L1026.

[71] Hess, D. C., Eldahshan, W. \& Rutkowski, E. (2020) COVID-19-Related Stroke. Transl. Stroke Res., 11, 322-325.

[72] Shovlin, C. L., \& Vizcaychipi, M. P. (2020) Vascular Inflammation and Endothelial Injury in SARS-CoV-2 Infection: The Overlooked Regulatory Cascades Implicated by the ACE2 Gene Cluster, QJM: An International Journal of Medicine, hcaa 241.

[73] Al-Quteimat, O. M., \& Amer, A. M. (2020) The Impact of the COVID-19 Pandemic on Cancer Patients, American Journal of Clinical Oncology, 43 (6), 452-455.

[74] Callaly, E. (2020) The Coronavirus is mutating-does it matter? Retrieved https://www.nature.com/articles/d41586-020-02544-6.

[75] Thermofisher Scientific (2021) Coronavirus mutations and variants-what do we know? Retrieved from, https://www.thermofisher.com/ng/en/home/clinical/clinical-ge nomics/pathogen-detection-solutions/covid-19-sars-cov-2/mut ations

variants.html?cid=gsd_cbu_sbu_r01_co_cp1422_pjt6968_gsd 00000 0se gaw ta lon em-global\&gclid=CjwKCAiAyc2BB hAaEiwA44-wW' $\overline{\text { ymib4 }}$ HJSEiDi017ZZTiLvIwohRCs9oFtdA R01JmVcoHWs9kopcPMBoCaO4QAvD_BwE. 
[76] U.S. Food and Drug Administration (2021) Genetic Variants of SARS-CoV-2 May Lead to False Negative Results with Molecular Tests for Detection of SARS-CoV-2 - Letter to Clinical Laboratory Staff and Health Care Providers, Retrieved from,

https://www.fda.gov/medical-devices/letters-health-care-provi ders/genetic-variants-sars-cov-2-may-lead-false-negative-resul ts-molecular-tests-detection-sars-cov-2.

[77] Li, Q., Guan, X., Wu, P., Wang, X., Zhou, L., Tong, Y., et al. (2020) Early Transmission Dynamics in Wuhan, China, of Novel Coronavirus Infected Pneumonia, N Engl J Med, 382 (13), 1199-1207.

[78] Chen, F., Liu, Z. S., Zhang, F. R., Xiong, R. H., Chen, Y., Cheng, X. F., et al. (2020) First Case of Severe Childhood
Novel Coronavirus Pneumonia in Chin, Zhonghua Er Ke Za Zhi, 58 (3), 179-182.

[79] de Souza, T. H., Nadal, J. A, Nogueira, R. J. N. Pereira, R. M., Brandão, M. B. (2020) Clinical Manifestations of Children with COVID-19: A Systematic Review. Pediatric Pulmonology. 55 (8), 1892-1899.

[80] Zainab, S., Ricci, K., Brendan, M., Douglas, K., Devyani, R., Ravi, P., Chander, A., Ramarao, V., Nitasa, S., Dhirisha, B., Kirk, J., Reshma, G., \& Rohit, J. (2020). Covid-19 and Older Adults: What We Know, Journal of the American Geriatrics Society, 68 (5), 926-929. 